\title{
Inhibiting wear particles-induced osteolysis with naringin
}

\author{
Xiaowei Yu • Xingwei Zhao • Tianyi Wu • \\ Zubin Zhou • Youshui Gao • Xinfu Wang • \\ Chang-Qing Zhang
}

Received: 24 July 2012 / Accepted: 16 September 2012 /Published online: 31 October 2012

(C) Springer-Verlag Berlin Heidelberg 2012

\begin{abstract}
Purpose The purpose of this study was to determine the effects of naringin on osteoclastogenesis and osteolysis both in vitro and in vivo.

Methods In this research osteoclasts were generated from mouse bone marrow monocytes with the receptor activator of NF-KB ligand and the macrophage colony stimulating factor. Naringin, at a concentration of $1,10,50$, and $100 \mu \mathrm{g} /$ $\mathrm{mL}$, was respectively added to the medium. Seven days later, the osteoclasts were determined through tartrateresistant acid phosphatase (TRAP) staining. Mature osteoclasts were isolated from newborn rabbits and cultured for three days on bone slices. Naringin at a concentration of 1 , 10,50 , and $100 \mu \mathrm{g} / \mathrm{mL}$ was respectively added to the medium. The resorption bone slices were quantified, and the area was calculated after toluidine blue and Mayerhematoxylin staining. Polymethyl methacrylate (PMMA) particles were implanted on the calvariae of $\mathrm{C} 57 \mathrm{BL} / \mathrm{J} 6$ mice. Naringin, at a dose of $50 \mu \mathrm{g} / \mathrm{kg}$ and $100 \mu \mathrm{g} / \mathrm{kg}$, was respectively given intraperitoneally for seven. Seven days later, the calvariae were removed and processed for pathological analysis.
\end{abstract}

Xiaowei $\mathrm{Yu}$ and Xinwei Zhao contributed equally as the first author.

X. Yu $\cdot$ Z. Zhou $\cdot$ Y. Gao $\cdot$ X. Wang $\cdot$ C.-Q. Zhang $(\bowtie)$

Department of Orthopaedics, Shanghai Sixth People's Hospital,

Shanghai Jiaotong University,

Shanghai, China

e-mail: zhangchangq@yahoo.com.cn

T. Wu

Department of Orthopaedics,

The Second Affiliated Hospital of Nanjing Medical University,

Nanjing, Jiangsu Province, China

X. Zhao

Department of Orthopaedics, Liao Cheng People's Hospital,

Liao Cheng, Shandong Province, China
Results The result indicated that naringin treatment effectively inhibited in vitro osteoclastogenesis and inhibited mature osteoclasts. In vivo data indicated that naringin strongly inhibited PMMA-induced osteolysis.

Conclusion Naringin can effectively inhibit osteoclastogenesis and suppress wear particles-induced osteolysis and might be useful in the treatment or prevention of wear particles-induced osteolysis and aseptic loosening for its effect on osteoclast generation and function.

\section{Introduction}

Total joint replacement is commonly used in clinics to treat severe arthritis affecting load-bearing joints for its effectiveness in reducing pain and improving joint function [1]. However, wear particles resulting from the degradation of artificial implantation may greatly influence the survival of joint arthroplasty [2]. Furthermore, wear particles may lead to osteolysis and are considered one of the principal contributors to prothesis aseptic loosening which may lead to consequent joint revision operation after total joint arthroplasty [3]. In detail, wear debris stimulates macrophage and other inflammatory cells to produce inflammatory cytokines, such as interleukin (IL)-1, IL-6, and TNF- $\alpha$ [4]. These inflammatory cytokines stimulate osteoblasts and stromal cells to produce large amounts of receptor activator of NF-KB ligand (RANKL) [5-7], a key player in initiating enhanced osteoclastogenesis and osteolysis.

Moreover, the mouse model of osteolysis even confirmed the central role of inflammatory cytokines, such as TNF- $\alpha$ and IL-1, in wear particles-induced osteolysis $[8,9]$. As a further step, osteoclastogenesis and the activation of osteoclasts lead to peri-prosthetic osteolysis and implant aseptic loosening [10]. Therefore, wear particles may cause adverse biological reactions in the following order: inflammation, 
increased osteoclastogenesis, and subsequent osteoclastic bone resorption [11]. Since osteoclastogenesis and osteoclast activation play essential roles in wear particles-induced osteolysis, inhibiting osteoclastogenesis and osteoclast function should be an important therapeutic strategy for the treatment of aseptic loosening after total joint replacement $[12,13]$.

In China, rhizoma drynariae has been widely used to manage orthopaedic disorders, and its therapeutic effects on bone metabolism and bone healing has been verified [14]. Current research on rhizoma drynariae extract indicated that it can enhance the proliferation and osteogenic differentiation of the MC3T3-E1 cell line [15] and inhibit osteoclast formation [16]. Based on the above, it seems that rhizoma drynariae has the potential therapeutic function for osteolysis-induced aseptic loosening. In the following research, naringin, a polymethoxylated flavonoid and the main effective component of rhizoma drynariae, was used and the inhibition of osteolysis after naringin management was confirmed.

\section{Materials and methods}

Inhibiting in vitro osteoclastogenesis with naringin

Osteoclasts were generated according to the research of Yasuda et al. [17]. Briefly, ten-week-old male C57BL/J6 mice were euthanized by sodium pentasorbital $(3 \%)$ and bone marrow cells were collected from femoral and tibial shafts by flushing with $8 \mathrm{~mL}$ cold Modified Eagle Medium (MEM, Gibco, Invitrogen, Grand Island, NY, USA) containing $10 \%$ Fetal Calf Serum FCS (Hyclone, Tauranga, New Zealand), $1 \%$ penicillin and streptomycin (Gibco, Invitrogen, Grand Island, NY, USA). Plastic-adherent bone marrow cells were removed by incubating at $37{ }^{\circ} \mathrm{C}$ with $5 \%$ $\mathrm{CO}_{2}$ in a humidified incubator for 24 hours. The bone marrow monocytes were isolated from the non-adherent cells in the supernatant by using Ficoll (The Second Chemical Reagent Factory of Shanghai, Shanghai, China). The bone marrow monocytes were cultured in the continuous presence of both macrophage colony-stimulating factor (MCSF, $30 \mathrm{ng} / \mathrm{mL}$; Research Diagnostics, Flanders, NJ, USA) and recombinant soluble RANKL (100 ng/mL; Research Diagnostics, USA) for seven days. The culture medium was changed every 48 hours with fresh M-CSF and RANKL. Naringin (N1376; from citrus fruit; chemical purity N90\%) was purchased from Sigma-Aldrich (St. Louis, MO) and was added when the M-CSF and RANKL were added and continued for seven days, with a final concentration of 1, 10, 50 and $100 \mu \mathrm{g} / \mathrm{mL}$, respectively. Seven days later, the cells were washed with phosphate buffer solution (PBS) and fixed in $2.5 \%$ glutaraldehyde for five minutes at room temperature. Tartrate-resistant acid phosphatase
(TRAP) staining (Shanghai Rainbow Medical Reagent Research, Shanghai, China) were performed and these multinucleate TRAP $(+)$ cells in the well were counted.

Inhibiting mature osteoclast function with naringin

Mature osteoclasts were isolated from seven-day-old New Zealand white rabbits (Shanghai SLAC Laboratory Animal, China) as previously reported [18]. Briefly, after sacrification with $250 \mathrm{mg}$ of pentobarbital, the rabbits were sterilized in $75 \%$ ethanol for five minutes and long bones were taken out and soft tissues attached to bones were removed. The bones were minced in MEM containing $10 \%$ FCS plus $1 \%$ penicillin and streptomycin. The cells were dissociated from the bone fragments by vortexing for 30 seconds and the fragments were allowed to sediment for one minute. The cells in the supernatant were collected and used as unfractionated bone cells. About $5 \times 10^{6}$ bone marrow cells were seeded into one well of the 24-well plate and incubated at $37{ }^{\circ} \mathrm{C}$ with $5 \% \mathrm{CO}_{2}$ for three days. For the bone resorption assay, $5 \times 10^{6}$ bone marrow cells were seeded onto $5 \times 5 \mathrm{~mm}$ bovine bone slices with the thickness of $0.5 \mathrm{~mm}$. Naringin was added to the culture medium at a final concentration of 1 , 10,50 , and $100 \mu \mathrm{g} / \mathrm{mL}$ respectively. Three days later, the cells were washed with PBS and fixed in $2.5 \%$ glutaraldehyde for five minutes at room temperature. After fixation, the cells on the bone slices were washed off with $25 \%$ ammonium hydroxide and sonicated three times for five minutes. The bone slices were then dehydrated through an ethanol gradient and stained with $1 \%$ toluidine blue and Mayer's hematoxylin. Positively stained areas were counted as bone resorption pits and the resorption area was calculated with Image Pro-Plus 5.0 (Media Cybernetics, Bethesda, MD, USA).

Inhibiting wear particles induced in vivo osteolysis with naringin

A wear-induced calvarial osteolysis model was prepared according to Schwarz EM's published paper [19]. Briefly, polymethyl methacrylate (PMMA) particles were applied to eight-week-old healthy C57BL/J6 male mice (Shanghai SLAC Laboratory Animal Co, China) according to the official guidelines of the Shang Hai Jiao Tong University (Shang Hai, China). The weight of the mice was $24 \pm 1 \mathrm{~g}$. Two doses of naringin $(50 \mu \mathrm{g} / \mathrm{kg}, 100 \mu \mathrm{g} / \mathrm{kg})$ were selected. Then, the animals were randomly classified into three groups with ten mice in each group: (1) control group, the mice received $30 \mathrm{mg}$ PMMA particle implantation; (2) PMMA+50ug naringin group, the mice received $30 \mathrm{mg}$ PMMA particles plus $50 \mu \mathrm{g} / \mathrm{kg}$ naringin intraperitoneal injection every day from day one to seven; (3) PMMA+ $100 \mathrm{ug}$ group, the mice received $30 \mathrm{mg}$ PMMA particles plus $100 \mu \mathrm{g} / \mathrm{kg}$ naringin intraperitoneal injection every day from 
day one to seven. The animals were kept in a specific pathogenfree animal room and had free access to water and food.

The PMMA particles and the osteolysis model were prepared according to Zhang C's published paper [4]. In detail, PMMA particles had a mean diameter of $2.6 \mathrm{~mm}$ (range, $0.6-21 \mathrm{~mm}$ ) and were suspended in sterile saline. Seven days later, the mice were killed with an overdose of anaesthesia and the calvariae were removed. Soft tissue of the specimens was removed and fixed in $4 \%$ neutralized paraformaldehyde for 12 hours and decalcified in $12.5 \%$ EDTA (pH 7.4) at $4{ }^{\circ} \mathrm{C}$ for three weeks. The decalcification solution was changed every three days. Then, graded ethanol for dehydration was performed and embedded in paraffin. Every specimen was sectioned on the sagittal plane with a 7- $\mu \mathrm{m}$ thickness (Leica RM2016). For the middle suture osteolysis area analysis, five consecutive sections of each specimen were stained with hematoxylin-eosin (Shanghai Rainbow Medical Reagent Research, China). Photos were taken at a magnification of $10 \times$ using Nikon Eclipse $80 \mathrm{i}$ microscope (Nikon, Tokyo, Japan). The non-osseous tissue area adjacent and in continuity with the midline suture was taken as the osteolysis of the calvarial bone and calculated with Image Pro-Plus 5.0 (Media Cybernetics, USA). To evaluate the osteoclastogenesis, the sections were stained using a commercial leukocyte acid phosphate staining kit (Shanghai Rainbow Medical Reagent Research, China). Red-stained cells in the middle suture and adjacent nonosseous osteolsysis area were counted as TRAP $(+)$ osteoclasts. Five horizons was randomly selected, the mean value was recorded as the number of TRAP $(+)$ osteoclasts in the section.
Fig. 1 TRAP staining after osteoclast induction for 7 days. RANKL and M-CSF induced a large number of osteoclasts, while effectively inhibiting osteoclastogenesis $(\times 10)$. a Control. b, c, d, e Naringin at 1 , 10,50 , and $100 \mu \mathrm{g} / \mathrm{mL}$, respectively. f The PMMA particles used in this research. $\mathbf{g}$ Large number of osteoclast formed after osteoclastogenic induction. Naringin effectively suppressed osteoclastogenesis.

* Significant difference compared with the control $(P<0.05$, $n=6$ )
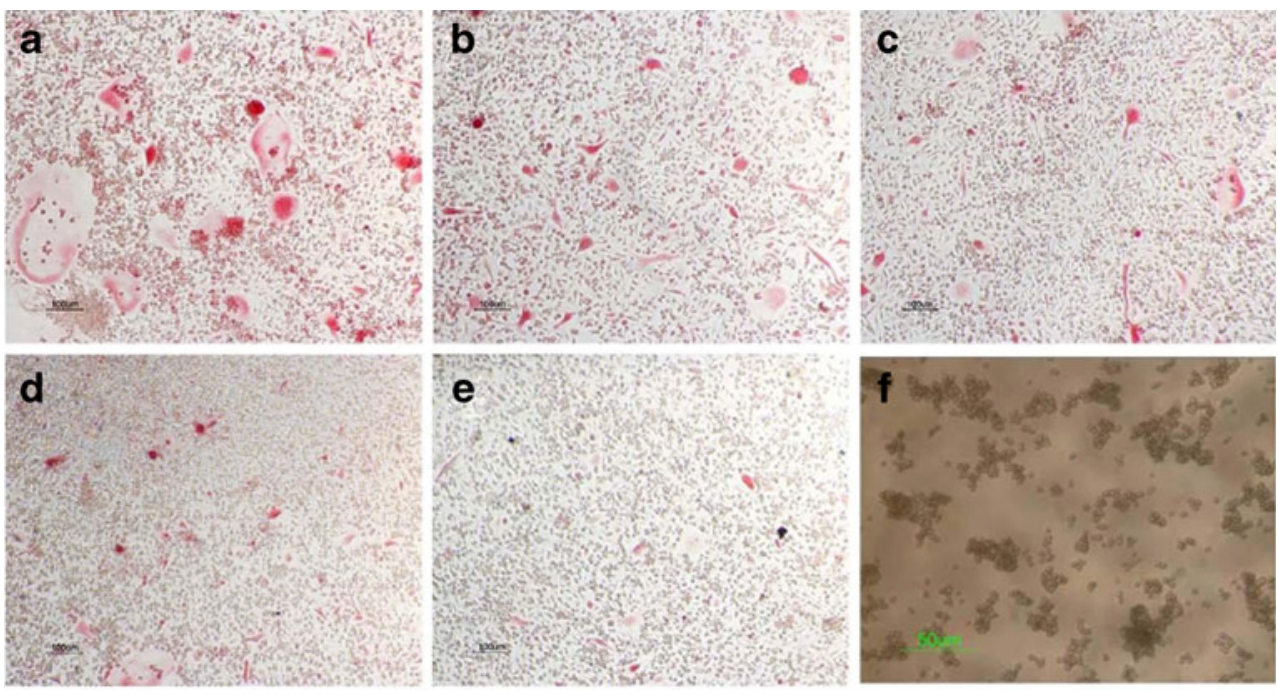

g

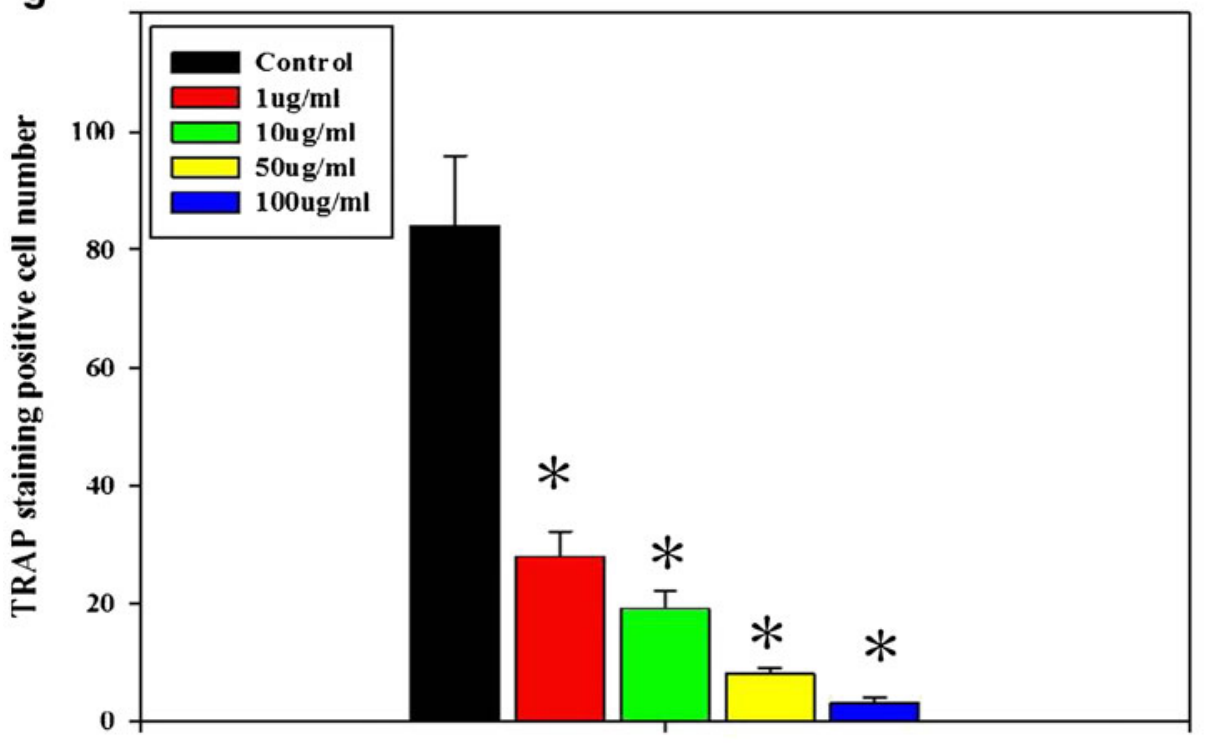

In vitro osteoclastogenesis inhibition 
Statistical analysis

All results were expressed as mean $\pm \mathrm{SD}$ and analysed by ANOVA, followed by Student's $t$-test to determine significance. $P<0.05$ was considered significant.

\section{Results}

Naringin suppressed osteoclastogenesis in vitro

Multinucleated cells (with more than three nuclei) formed after using bone marrow osteoclast precursors induced with RANKL and M-CSF (Fig. 1a). Naringin applied at a concentration of $1 \mu \mathrm{g} / \mathrm{mL}$, significantly inhibited osteoclastogenesis induced by RANKL and M-CSF, with a dose dependent manner from $1 \mu \mathrm{g} / \mathrm{ml}$ to $100 \mu \mathrm{g} / \mathrm{mL}(P<0.05$; Fig. 1b-e, g).
Naringin suppressed mature osteoclast bone resorption

After culture in DMEM on bovine cortical bone slices for three days, the bone resorption pits were counted and the resorption area was measured. The formation of resorption lacuna was found after staining with toluidine blue and Mayer-hematoxylin (Fig. 2a). Generally, resorption pit number was greatly reduced with the increasing of naringin concentration (Fig. 2b-e). The resorption pit number was about $124.3 \pm 18.4$ in the control. However, the naringin at a concentration of $1 \mu \mathrm{g} / \mathrm{ml}$ significantly reduced the resorption lacunae number to $82 \pm 15.8$. With the increase of naringin concentration $(10 \mu \mathrm{g} / \mathrm{ml}, 50 \mu \mathrm{g} / \mathrm{ml}, 100 \mu \mathrm{g} / \mathrm{ml})$, the resorption pit number decreased accordingly, with significant difference compared with the control $(P<0.05$; Fig. 2f). The resorption area measurement also showed that naringin significantly reduced the resorption area on cortical slices.
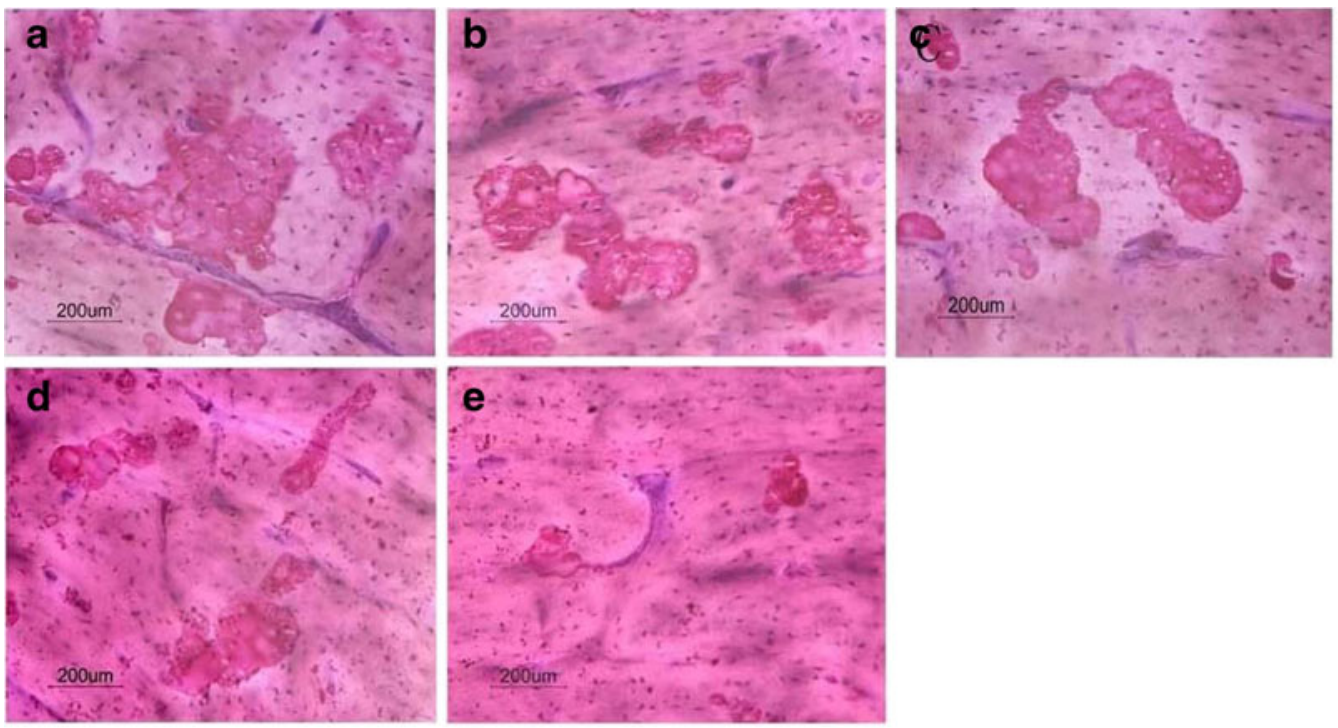

f

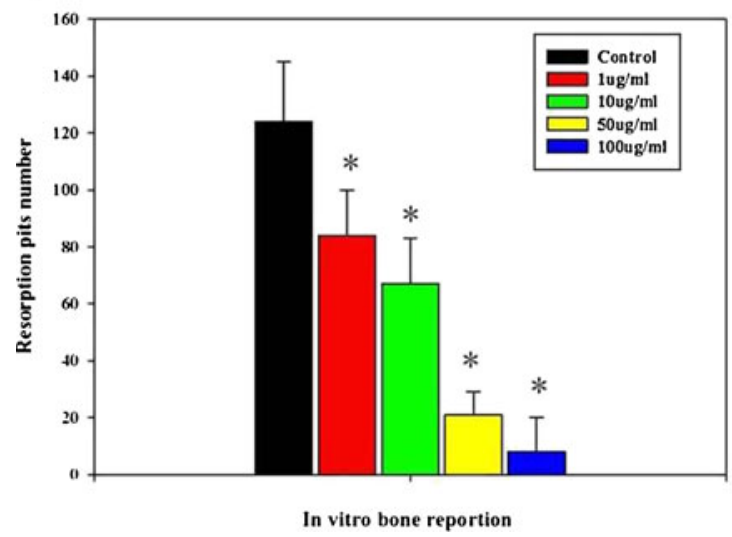

Fig. 2 Naringin inhibit rabbit mature osteoclasts' resorption on bone cortical slices (a-e Control, $1 \mu \mathrm{g} / \mathrm{ml}, 10 \mu \mathrm{g} / \mathrm{ml}, 50 \mu \mathrm{g} / \mathrm{ml}, 100 \mu \mathrm{g} / \mathrm{ml}$, respectively). Toluidine blue-Mayer's hematoxycycline staining showed the formation of a lot of resorption pits in the control group; g

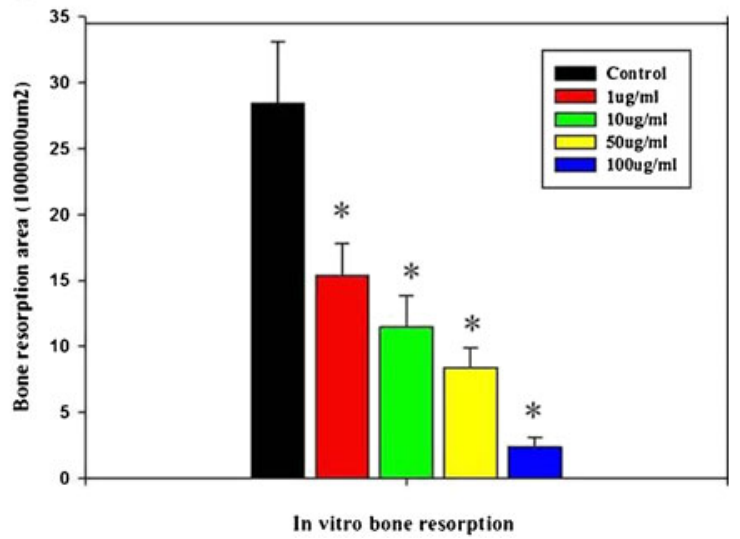

naringin treatment significantly reduced the number of resorption pits (Fig. 3f). Resorption pits area analysis showed that the total resorption area was effectively inhibited by naringin $(\mathbf{g}) .{ }^{*}$ Significant difference compared with the control $(P<0.05, n=6)$ 
In the control, the resorption was $(15.4 \pm 2.3) \times 10^{6} \mu \mathrm{m}^{2}$. After adding naringin to the culture medium with concentration of $10 \mu \mathrm{g} / \mathrm{ml}, 50 \mu \mathrm{g} / \mathrm{ml}$, and $100 \mu \mathrm{g} / \mathrm{ml}$, the resorption area was reduced into $(11.2 \pm 2.2) \times 10^{6} \mu \mathrm{m}^{2},(8.4 \pm 1.5) \times$ $10^{6} \mu^{2}$, and $(2.4 \pm 0.7) \times 10^{6} \mu \mathrm{m}^{2}$, respectively. With significant difference compared with the control $(P<0.05$; Fig. 2g).

Naringin inhibited in vivo osteolysis caused by wear particles

\section{Osteoclast number}

The osteoclast number within the experimental areas in the control group was $25.2 \pm 3.9$ (Fig. 3b). While in the
PMMA $+50 \mu \mathrm{g} / \mathrm{kg}$ naringin group the TRAP $(+)$ number was $9.3 \pm 2.1$ (Fig. 3c), in the PMMA $+100 \mu \mathrm{g} / \mathrm{kg}$ naringin group it was $4.1 \pm 1.3$. (Fig. 3d), indicating strongly suppressed osteoclastogenesis induced by PMMA after using naringin (Fig. 3e).

\section{Sagittal suture resorption area}

The sagittal suture resorption area in the control group was $0.545 \pm 0.082 \mathrm{~mm}^{2}$ (Fig. 3b). In the PMMA $+50 \mu \mathrm{g} / \mathrm{kg}$ naringin group, the mean sagittal suture area was $0.341 \pm$ $0.073 \mathrm{~mm}^{2}$, and in the PMMA $+100 \mu \mathrm{g} / \mathrm{kg}$ naringin group, the area was $0.239 \pm 0.029 \mathrm{~mm}^{2}$ with significant difference compared with the control $(P<0.05$, Fig. 3f).
Fig. 3 a In vivo mouse calvarial osteolysis sample. b, c, d PMMA induced bone resorption and the osteoclastogenesis (TRAP stain) in the control group, the $50 \mu \mathrm{g} / \mathrm{kg}$ group and the 100 $\mu \mathrm{g} / \mathrm{kg}$ group. e, f The osteolysis area calculation and the TRAP staining positive cell count in three groups (control, $50 \mu \mathrm{g} / \mathrm{kg}$, $100 \mu \mathrm{g} / \mathrm{kg})$. *Significant difference compared with the control group $(P<0.05, n=6)$
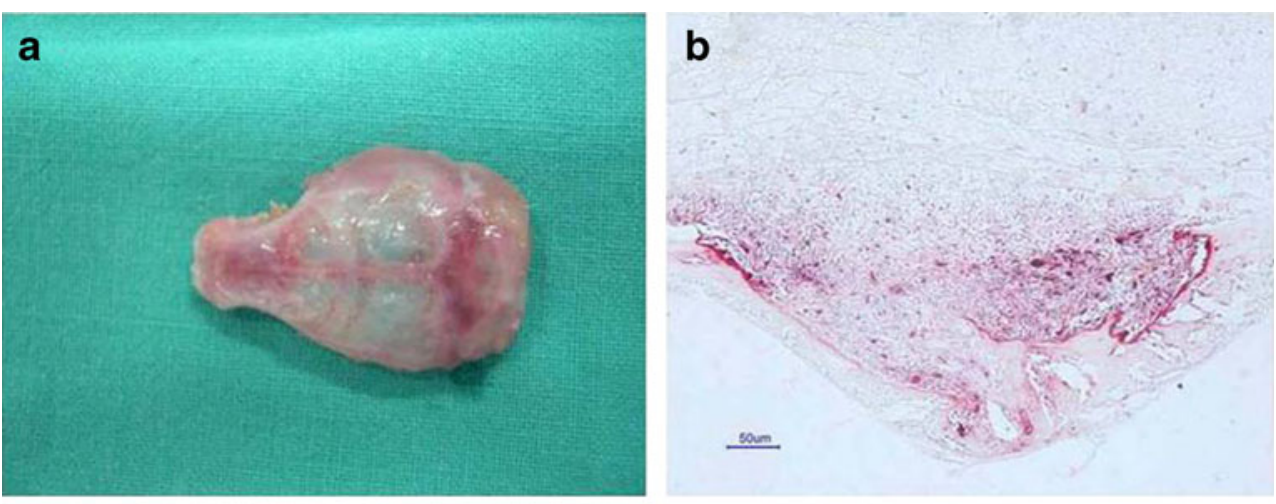

C

d
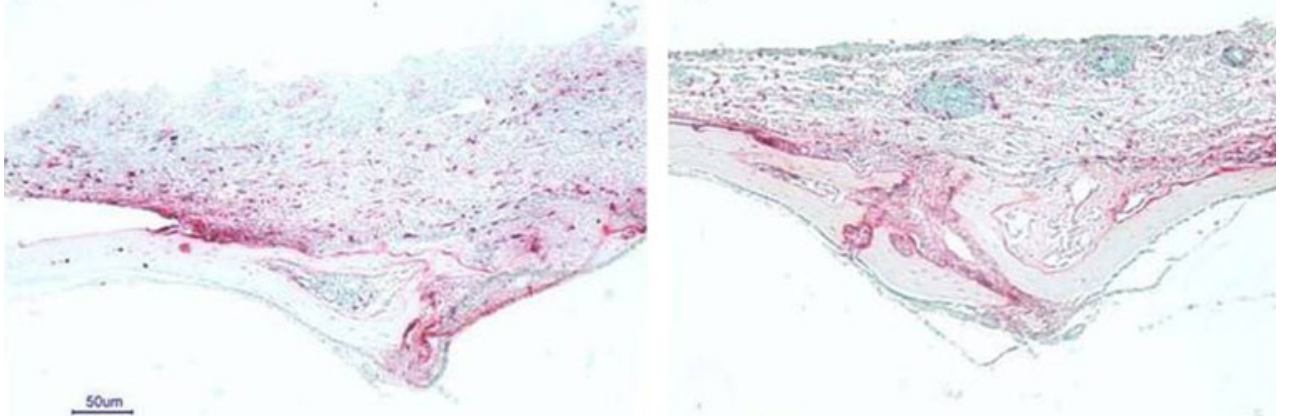

e

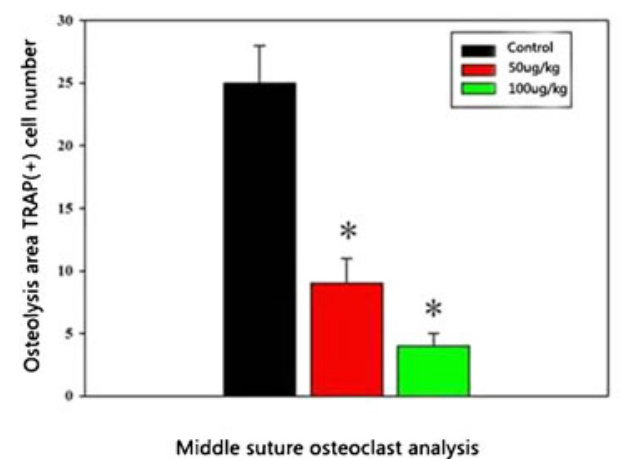

f

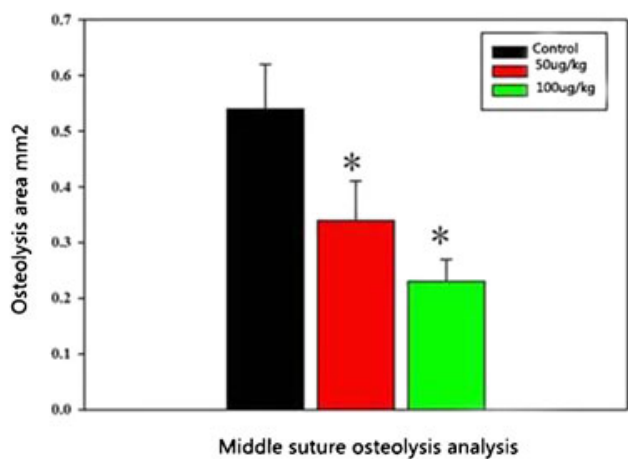




\section{Discussion}

Aseptic loosening-induced artificial joint failure greatly influences the long-term result of total joint replacement $[3,10,20]$. Current research shows that osteoclast is the centre and final pathway of wear particles-induced osteolysis. Therefore, inhibiting osteoclastogenesis is of great importance. Actually, many efforts have been made for the treatment of wear particle-induced osteolysis [21, 22]. Etanercept, a TNF- $\alpha$ receptor antagonist and bisphosphonate, has been applied in clinics after total joint arthroplasty [23-25].

However, these methods have different side effects; therefore, it is necessary to find efficient and affordable methods for the treatment of aseptic loosening. It is a viable method to find effective pharmaceutical ingredients from traditional Chinese medicine. In traditional Chinese medicine, many herbs have been used for thousands of years with sufficient proof of evidence-based medicine for the treatment of osteoporosis and bone nonunion to strengthen bone quality. Hence, many herbs and effective ingredients should be selected for the treatment of osteolysis-induced aseptic loosening.

Following this principle, in this paper, naringin, which is the main effective ingredient of rhizoma drynariae (a kidneytonifying herbal), was used to confirm its inhibition to the osteoclastogenensis and osteolysis. According to literature review, Zhang et al. have proven the osteogenic induction function of naringin [26]. From another side, our research proved the bone protective effect of naringin in view of inhibition of osteolysis.

In conclusion, for the first time, our study proved the inhibition of osteoclastogenesis and osteolysis using naringin in vitro and in vivo, which indicates that naringin has the potential therapeutic function to be used for the treatment of aseptic loosening. It should be mentioned that, besides our current research, there are more issues which should be verified in the future. First, in-depth research about the molecular mechanism by which naringin influences the osteoclastogenesis should be performed. Then, besides the intraperitoneal injection, the therapeutic effect of other ways of medicating, such as oral administration, intramuscular injection, etc. should be further confirmed.

Acknowledgments This research was supported by the Shanghai Postdoctoral Sustentation Fund, China (Grant No. 11R21414400), China Postdoctoral Science Foundation (Grant No. 20100480598) and National Natural Science Foundation of China (Grant No. 81071487).

Conflicts of interest The authors declare that they have no conflict of interest with this paper.

\section{References}

1. Huo MH, Dumont GD, Knight JR, Mont MA (2011) What's new in total hip arthroplasty. J Bone Joint Surg Am 93:1944-1950

2. Billi F, Campbell P (2010) Nanotoxicology of metal wear particles in total joint arthroplasty: a review of current concepts. J Appl Biomater Biomech 8:1-6

3. Kaddick C, Catelas I, Pennekamp PH, Wimmer MA (2009) Implant wear and aseptic loosening. An overview. Orthopade 38:690-697

4. Zhang C, Tang T, Ren W, Zhang X, Dai K (2008) Influence of mouse genetic background on wear particle-induced in vivo inflammatory osteolysis. Inflamm Res 57:211-215

5. Baumann B, Rader CP, Seufert J, Noth U, Rolf O, Eulert J et al (2004) Effects of polyethylene and TiAlV wear particles on expression of RANK, RANKL and OPG mRNA. Acta Orthop Scand 75:295-302

6. Koreny T, Tunyogi-Csapo M, Gal I, Vermes C, Jacobs JJ, Glant TT (2006) The role of fibroblasts and fibroblast-derived factors in periprosthetic osteolysis. Arthritis Rheum 54:3221-3232

7. Wooley PH, Morren R, Andary J, Sud S, Yang SY, Mayton L et al (2002) Inflammatory responses to orthopaedic biomaterials in the murine air pouch. Biomaterials 23:517-526

8. Epstein NJ, Warme BA, Spanogle J, Ma T, Bragg B, Smith RL et al (2005) Interleukin-1 modulates periprosthetic tissue formation in an intramedullary model of particle-induced inflammation. J Orthop Res 23:501-510

9. Zhou X, Zhang C, Wang X, An B, Zhang P, Zhu Z (2012) Berberine inhibits lipopolysaccharide- and polyethylene particleinduced mouse calvarial osteolysis in vivo. J Surg Res 173:e47e52

10. Zhang P, Qin L (2010) Potential use of erythromycin to prevent and treat prosthetic failure. J Clin Pharmacol 50:858-860

11. Catelas I, Jacobs JJ (2010) Biologic activity of wear particles. Instr Course Lect 59:3-16

12. Geng DC, Zhu XS, Mao HQ, Meng B, Chen L, Yang HL et al (2011) Protection against titanium particle-induced osteoclastogenesis by cyclooxygenase- 2 selective inhibitor. J Biomed Mater Res A 99:516-522

13. Markel DC, Zhang R, Shi T, Hawkins M, Ren W (2009) Inhibitory effects of erythromycin on wear debris-induced VEGF/Flt-1 gene production and osteolysis. Inflamm Res 58:413-421

14. Wong RW, Rabie B, Bendeus M, Hagg U (2007) The effects of Rhizoma Curculiginis and Rhizoma Drynariae extracts on bones. Chin Med 2:13

15. Jeong JC, Lee JW, Yoon CH, Lee YC, Chung KH, Kim MG et al (2005) Stimulative effects of Drynariae Rhizoma extracts on the proliferation and differentiation of osteoblastic MC3T3-E1 cells. J Ethnopharmacol 96:489-495

16. Jeong JC, Kang SK, Youn CH, Jeong CW, Kim HM, Lee YC et al (2003) Inhibition of Drynariae Rhizoma extracts on bone resorption mediated by processing of cathepsin $\mathrm{K}$ in cultured mouse osteoclasts. Int Immunopharmacol 3:1685-1697

17. Yasuda H, Shima N, Nakagawa N, Yamaguchi K, Kinosaki M, Mochizuki S et al (1998) Osteoclast differentiation factor is a ligand for osteoprotegerin/osteoclastogenesis-inhibitory factor and is identical to TRANCE/RANKL. Proc Natl Acad Sci USA 95:3597-3602

18. Sobue T, Hakeda Y, Kobayashi Y, Hayakawa H, Yamashita K, Aoki $\mathrm{T}$ et al (2001) Tissue inhibitor of metalloproteinases 1 and 2 directly stimulate the bone-resorbing activity of isolated mature osteoclasts. J Bone Miner Res 16:2205-2214 
19. Schwarz EM, Benz EB, Lu AP, Goater JJ, Mollano AV, Rosier RN et al (2000) Quantitative small-animal surrogate to evaluate drug efficacy in preventing wear debris-induced osteolysis. J Orthop Res 18:849-855

20. Goodman SB, Gomez Barrena E, Takagi M, Konttinen YT (2009) Biocompatibility of total joint replacements: a review. J Biomed Mater Res A 90:603-618

21. Ren W, Zhang R, Wu B, Wooley PH, Hawkins M, Markel DC (2011) Effects of SU5416 and a vascular endothelial growth factor neutralizing antibody on wear debris-induced inflammatory osteolysis in a mouse model. J Inflamm Res 4:29-38

22. Zhang W, Zhao H, Peng X, Cheng T, Zhang X (2011) Low-dose captopril inhibits wear debris-induced inflammatory osteolysis. J Int Med Res 39:798-804
23. Childs LM, Goater JJ, O'Keefe RJ, Schwarz EM (2001) Efficacy of etanercept for wear debris-induced osteolysis. J Bone Miner Res $16: 338-347$

24. Schwarz EM, Looney RJ, O'Keefe RJ (2000) Anti-TNF-alpha therapy as a clinical intervention for periprosthetic osteolysis. Arthritis Res 2:165-168

25. Tsutsumi R, Hock C, Bechtold CD, Proulx ST, Bukata SV, Ito H et al (2008) Differential effects of biologic versus bisphosphonate inhibition of wear debris-induced osteolysis assessed by longitudinal micro-CT. J Orthop Res 26:1340-1346

26. Zhang P, Dai KR, Yan SG, Yan WQ, Zhang C, Chen DQ et al (2009) Effects of naringin on the proliferation and osteogenic differentiation of human bone mesenchymal stem cell. Eur J Pharmacol 607:1-5 